PAPER

\title{
Magnetoencephalography (MEG) predicts focal epileptogenicity in cavernomas
}

\author{
H Stefan, G Scheler, C Hummel, J Walter, J Romstöck, M Buchfelder, I Blümcke
}

J Neurol Neurosurg Psychiatry 2004;75:1309-1313. doi: 10.1136/jnnp.2003.021972

See end of article for authors' affiliations ......................

Correspondence to: Prof. Dr med. H Stefan, Department of Neurology, Epilepsy and NeuroCenter, University of Erlangen-Nürnberg, Schwabachanlage 6 , D-91054 Erlangen, Germany; hermann.stefan@neuro. imed.uni-erlangen.de

Received 26 June 2003 In revised form

13 November 2003 Accepted

17 December 2003

\begin{abstract}
Objective: The aim of this study was to identify the irritative epileptic zone in patients with cavernomas by means of magnetoencephalography (MEG).

Method: Among 82 patients operated for epilepsy, whose presurgical evaluation had included MEG, histological assessment of the tissue removed had confirmed cavernomas in eight. These eight patients had epilepsy since 18.6 (SD 12.7) years on average. The monitoring lasted about 2.1 (SD 1.3) hours and a median 20.9 (SD 14.3) spikes per hour were recorded. Spontaneous brain activity was recorded by means of a 74 channel dual unit MEG system (Magnes II, 4-D Neuroimaging) with simultaneous EEG recording (31 scalp electrodes). Spike analysis was performed using different source (moving dipole, current density reconstruction) and head models (spherical shells, BEM). Co-registration of neurophysiological and imaging data (MRI) was based upon anatomical landmarks.

Results: In 6/8 patients co-localisation from the cavernoma and epileptic zone was found. In two patients the focus was localised in the parieto-occipital lobe, in three patients in the frontal lobe and in three patients in the temporal lobe. In one case of temporal and one case of frontal lobe focus localisation there was no spatial relationship to the cavernoma.

Conclusion: In cases of focal seizures due to a single cavernoma, MEG may precisely delineate the epileptogenic tissue bordering the lesion. In patients with multiple cavernomas or dual pathology, MSI may reveal the complexity of the case, and contribute to the decision about further invasive diagnostics and more sophisticated therapeutic measures. MEG is a promising method for prediction of the epileptic zone in cavernoma related epilepsies, and thus it can contribute to decision making about and planning of epilepsy surgery.
\end{abstract}

C avernous angiomas represent about $5-20 \%$ of all vascular malformations in the central nervous system. Multiple cavernomas may occur in $20-40 \%$ of affected patients. Magnetic resonance imaging (MRI) is the most appropriate method to identify cavernomas. Microhaemorrhages within and around the lesions are an integral diagnostic feature that can usually only be inferred from irregular haemosiderin deposits detectable in $\mathrm{T} 1$ and $\mathrm{T} 2$ weighted MR images.

Seizures are estimated to occur in $40-70 \%$ of patients with cavernomas. Most of the seizures are focal, without or with secondary generalisation. ${ }^{1}$ A number of different mechanisms have been proposed to cause epilepsy: mass effects on, gliosis of, or haemosiderin deposition in the surrounding brain tissue. Studies using neocortical seizure models indicated that the tissue adjacent to rather than within the lesion exhibits hyperexcitability and thus may be critical in seizure generation. Intracellular neuronal recordings showed that especially neocortical neurones in the vicinity of cavernous malformations had a greater propensity for large complex spontaneous synaptic events than neurones neighbouring tumours. These data also revealed that occurrence of these abnormalities was proportional to the proximity to the lesion, suggesting seizure production via a different mechanism than gliocytes. ${ }^{2}$

With only a minority of patients responding to antiepileptic drug (AED) treatment, and with a high bleeding risk of $0.7 \%$ per year, the indication for surgical treatment has become less restrictive in recent years.

In the case of pharmacoresistant epilepsies, several questions need to be answered before planning surgery:

- Does the cavernoma represent the site of epileptogenicity?
- What is the detailed spatial relationship between the lesion and the epileptogenic focus?

- Are there multiple cavernomas and/or multiple epileptic foci?

A second evaluation for the focus of epileptogenicity in cases with poor outcome after surgery is another challenge for diagnostic modalities or those situations which are complicated further by the severe alteration of features that are relevant for both electrophysiological evaluation and further neurosurgical procedures. ${ }^{3}$

Magnetoencephalography (MEG), a non-invasive tool for the assessment of focal epileptic activity, has been reported to accurately localise specific epileptic discharges in a variety of publications $^{4-9}$ and to be particularly useful in combination with MRI data-known as "magnetic source imaging" (MSI)..$^{10-17}$

Although MEG has been successfully applied in presurgical evaluation of lesional epilepsies, ${ }^{18-23}$ no systematic studies have as yet been conducted about the spatial relation between MEG localisation of spike activity, MRI localisation of lesions, histological examination, and surgical outcome in patients with cavernomas. Therefore, we conducted a study to investigate whether MEG spike localisations were able to locate the epileptic tissue in focal epilepsies associated with cavernomas.

\section{PATIENTS AND METHODS}

Among 82 patients operated for epilepsy, whose presurgical evaluation had included MEG, histological assessment of the

Abbreviations: $A E D$, antiepileptic drug; $E C D$, equivalent current dipole; MEG, magnetoencephalography; MRI, magnetic resonance imaging 
tissue removed had confirmed cavernomas in eight. These eight patients with pharmacoresistant focal epilepsies (six men, two women; mean age at presurgical evaluation 30.6 years, range 15-51) were included in the study. They had simple as well as complex partial seizures since an average 18.6 (SD 12.7) years. All but one had secondary generalised tonic-clonic seizures.

Two patients underwent lesionectomy for a histologically ascertained cavernoma in the left temporal lobe of cavernomas, but with poor outcome. Neither of them were examined during their first course of presurgical evaluation.

In addition to the routine diagnostic procedures during presurgical epilepsy evaluation, all patients underwent exploration with video electroencephalographic (EEG) monitoring (64 channels, Glonner Neurosys system; Glonner, Munich, Germany) MSI.

MEG was recorded with a 74 channel dual unit biomagnetometer (Magnes II, 4D-Neuroimaging, Aachen, Germany) with a $5 \mathrm{~cm}$ gradiometer baseline. Spontaneous magnetic activity was continuously recorded for the purpose of focus assessment. The patients were usually positioned lying on the side between the sensor units. Sitting upright or halfreclining positions were chosen for recording activity from central and midline regions, with the area of interest beneath the sensors, in single unit mode. The sensors were adjusted with respect to the presumed area of epileptogenicity. Monitoring lasted about an average of 2.1 (SD 1.3) hours and a median 20.9 (SD 14.3) spikes per hour were recorded.

The data were co-registered with the MRI pictures by a procedure based upon anatomical landmarks (nasion and preauricular points). These were digitised prior to the MEG recordings with a three dimensional digitiser (Polhemus, Colchester, VT) with relation to the MEG sensors and identified in the MR images by means of self adhesive radiographic markers. Reproducibility of the reference points was verified by digital photography. MRI data were available for all patients.

Continuous data were band pass filtered offline $(3-70 \mathrm{~Hz})$, and visually inspected for specific epileptic patterns to obtain the earliest possible dipole localisations. Standard evaluation was based upon the model of an equivalent current dipole
(ECD) in a homogeneously conducting sphere. The best fitting dipoles were selected by filtering the dipole results using a 0.98 map correlation and a $3 \mathrm{ml}$ confidence volume threshold. In addition, we are applying more sophisticated techniques in an ongoing study aiming at the comparison of MEG and EEG results using different strategies of analysis, including realistically shaped head models (boundary element method BEM and current density reconstructions (Curry software, Neuroscan).

\section{RESULTS}

Table 1 summarises the patients' data and results of the investigations.

In all eight patients MSI yielded distinct localisations of epileptic discharges. In 6/8 patients MEG could locate spike activity close to the cavernoma and precisely delineate the epileptogenic tissue bordering the lesion. These six patients underwent surgical treatment with the details of the therapeutic plan based upon the MSI findings. Among these six, five were treated with resective surgery according to MSI localisations, one of them with additional multiple subpial transsection (MST).

Magnetic source localisations of specific epileptic activity were generally found to be clustered rather than diffusely distributed. In all patients but one, dipole clusters showed close spatial relationship to lesions, with dipoles accumulated at circumscribed locations in the vicinity of the cavernomas or resection cavities rather than scattered around the lesions. The maximum distance between the border of the clusters and the border of the lesion was $20 \mathrm{~mm}$. In the last case (no. $5)$, localisation results indicated spike generation in an area remote from the lesion, with more than $3 \mathrm{~cm}$ between the border of the dipole cluster and the margin of the lesion. This patient had had surgery previously without presurgical MEG investigation. Since he had not benefited, with respect to seizures, from the initial removal of a cavernoma in the basal anterior temporal region, he underwent re-evaluation for epilepsy, this time including MSI. The MSI results revealed the centre of epileptic activity to be located in the superior posterior temporal region, clearly unrelated to the resection cavity (fig 1). The patient was finally treated with fractioned

Table 1 Clinical data and results of investigation for the eight study patients

\begin{tabular}{|c|c|c|c|c|c|c|c|c|}
\hline $\begin{array}{l}\text { Patient } \\
\text { no. }\end{array}$ & $\begin{array}{l}\text { Age of } \\
\text { onset of } \\
\text { seizures } \\
\text { (years) }\end{array}$ & Sex & $\begin{array}{l}\text { Sps/Cps/ } \\
\text { Sec tc }\end{array}$ & Lesion(s) & $\begin{array}{l}\text { Video-EEG } \\
\text { monitoring } \\
\text { focus result }\end{array}$ & $\begin{array}{l}\text { MSI max. dist. } \\
\text { to lesion* }\end{array}$ & $\begin{array}{l}\text { Treatment } \\
\text { (final) }\end{array}$ & $\begin{array}{l}\text { Follow } \\
\text { upt }\end{array}$ \\
\hline 1 & 13 & $\mathrm{~F}$ & $Y$ & 1: Le T & Le Fr T & $1:<2 \mathrm{~cm}$ & ext. $\mathrm{LE}+\mathrm{HE}$ & $1 \mathrm{~A}$ \\
\hline 2 & 11 & $\mathrm{~F}$ & Y & $\begin{array}{l}\text { 1: Le T, res } \\
\text { 2: Le P } \\
\text { 3: Ri F }\end{array}$ & Le $\mathrm{T}>\mathrm{Ri} \mathrm{T}$ & $\begin{array}{l}\text { 1: Le } \mathrm{T}<2 \mathrm{~cm} \\
\text { 2: Le } \mathrm{P}<2 \mathrm{~cm} \\
\text { 3: } \varnothing\end{array}$ & $\begin{array}{l}\text { 1: LE+ext. } \\
\text { 2: MST }\end{array}$ & $3 A$ \\
\hline 3 & 23 & $M$ & $Y$ & 1: Le T & Le T & $1:<1 \mathrm{~cm}$ & ext. $\mathrm{LE}+\mathrm{HE}$ & 1B \\
\hline 4 & 6 & M & $Y$ & $\begin{array}{l}\text { 1: Le PO } \\
\text { 2: Le O }\end{array}$ & Le T P O & $\begin{array}{l}\text { 1: Le } \mathrm{P} \mathrm{O}<1 \mathrm{~cm} \\
2: \varnothing\end{array}$ & $\mathrm{LE}$ & $4 \mathrm{~B}$ \\
\hline 5 & 14 & M & $Y$ & 1: Le $\mathrm{T}$, res & Le $T>$ Ri T & $1:>3 \mathrm{~cm}$ & foc. st. RdT & $3 A$ \\
\hline 6 & 20 & M & $Y$ & $\begin{array}{l}\text { 1: Le T } \\
\text { 2: Re Fr }\end{array}$ & Ri Fr T $>$ Li Fr T & $\begin{array}{l}\text { 1: Le } T<1 \mathrm{~cm} \\
2: \varnothing\end{array}$ & Le T tail. res. & $2 A$ \\
\hline 7 & 20 & $M$ & $Y$ & 1: Le Fr & Le, Ri Fr T & $1:<1 \mathrm{~cm}$ & ext. LE & $1 \mathrm{~A}$ \\
\hline 8 & 11 & M & Y & $\begin{array}{l}\text { 1: Ri Fr } \\
\text { 2: Ri T } \\
\text { 3: Ri O } \\
\text { 4: Le O }\end{array}$ & Le, Ri Fr T & $\begin{array}{l}\text { 1: } \operatorname{Ri~} \mathrm{Fr}<1 \mathrm{~cm} \\
\text { 2: } \operatorname{Ri} \mathrm{Fr}>3 \mathrm{~cm} \\
\text { 3: } \varnothing \\
4: \varnothing\end{array}$ & none & $" 4 B^{\prime \prime}$ \\
\hline \multicolumn{9}{|c|}{$\begin{array}{l}\text { *Max. dist. to lesion, maximum distance between dipole cluster and margin of lesion, with the site of lesion } \\
\text { specified in case of multiple lesions. } \\
\text { tFollow up: according to Engel's classification. } \\
\varnothing \text {, no relationship to lesion. } \\
\text { cps, complex partial seizures; EEG, electroencephalography; ext., extended; } F \text {, female; Fr, frontal; foc. st. RdT, } \\
\text { focal stereotactic radiotherapy; HE, hippocampectomy; Le, left; LE, lesionectomy; } M \text {, male; MST, multiple } \\
\text { stereotactic transsection; N, no; O, occipital; P, parietal; res, resection cavity after previous removal of a } \\
\text { cavernoma; Ri, right; sec tc, secondary tonic clonic seizures; sps, simple partial seizures; tail. res., tailored } \\
\text { resection according to intraoperative ECoG; T, temporal; } Y \text {, yes. }\end{array}$} \\
\hline
\end{tabular}


low dose radiotherapy, ${ }^{24}$ which markedly improved his seizure situation.

Analysis of the spatial relation between MEG spike sources and lesions with respect to surgical outcome revealed that if the distance between the localisation of epileptic activity and the lesion was less than $20 \mathrm{~mm}$, if there was just one cavernoma, and if the respective tissue could be included in the resected volume, prognosis of seizure control after resection was favourable (fig 2). Success with respect to seizure control was independent of the lobe of epileptogenesis. Figure 3 shows a case of symptomatic epilepsy due to a cavernoma in the frontal lobe.

Four patients had multiple cavernomas (case nos 2, 4, 6, 8). In all four cases, MSI revealed the centres of epileptic activity and their relationship to the lesions. In two of the patients (case nos 4,6 ), one of the cavernomas was singled out as the epileptogenic lesion. In both cases, the critical lesion was removed. One patient (no. 4) did not benefit from the treatment with respect to seizures because the cavernoma was within the visual cortex so only the cavernoma was resected. The second patient (no. 6) experienced major improvement after surgery. In the remaining two patients multiple foci related to lesions were identified. One of them (no. 2) presented residual epileptic activity after removal of a cavernoma in the left temporal lobe. Dipole clusters were closely related to both the remaining cavernoma in the left parietal region and the temporal resection cavity, whereas the third cavernoma in the right frontal lobe showed no epileptogenicity. This patient was finally treated with an extension of the temporal resection and parietal MST, but the outcome was only moderate. Patient no. 8 had four cavernomas: one in the left occipital lobe and three in the right hemisphere, in the frontal, temporal, and occipital lobes. MSI revealed epileptic activity on the border of the frontal lesion and, in addition, in the frontopolar region with no relationship to any lesion. These findings and the various lesions are shown in fig 4 . The patient has not been treated surgically. We classified his outcome as analogous to Engel classification " $4 \mathrm{~B}$ ", even without surgical treatment.

\section{DISCUSSION}

In all patients the MSI results indicated particular sites at the margins of the lesions to generate epileptic spikes-in six patients with a maximum of $20 \mathrm{~mm}$ between the border of dipole locations and the border of the lesion. These results in patients with cavernomas indicate that MEG is a potent tool for establishing a detailed localisation of epileptogenicity of cavernomas and their surrounding tissue. This is in concordance with other studies of lesional epilepsies which

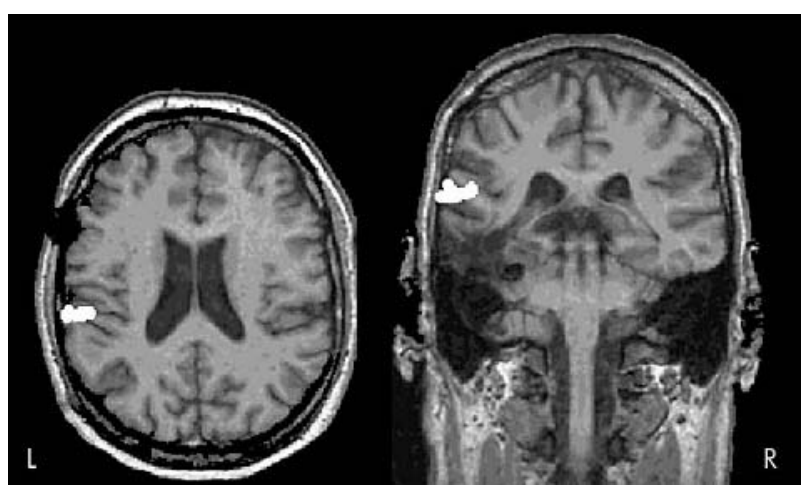

Figure 1 Patient with persisting seizures after resection of a cavernoma. Postoperative MEG spike localisations (white dots), unrelated to resection cavity. Source model: moving dipole; volume conductor model: sphere.

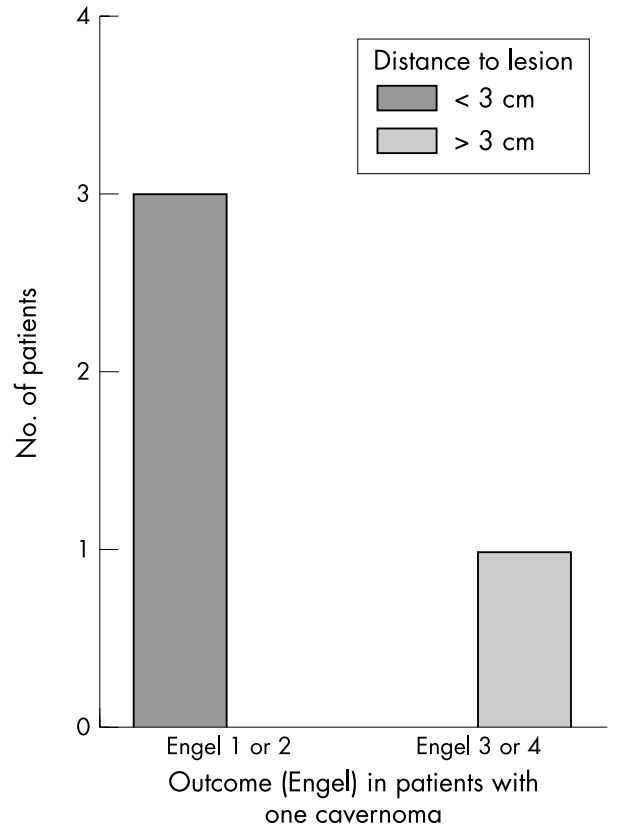

Figure 2 Outcome in patients with one cavernoma. Dark grey: the outcome in these three patients was 1A, 1B and 2A (Engel classification at least six months after surgery) with the lesion to resection distance less than $3 \mathrm{~cm}$. Light grey: in this patient the outcome classification (Engel) was $3 \mathrm{~A}$, and distance to the lesion more than $3 \mathrm{~cm}$.

found that MEG dipole clusters are often restricted to one side of the respective lesions. ${ }^{1422}{ }^{25}$ MSI contributes to the prognosis of subsequent surgery which can be promising if the epileptogenic tissue is localised immediately adjacent to the cavernoma. Similar to the findings of Awad and coworkers $^{18}$ who investigated the relationship of epileptogenic tissue and structural brain lesions using intracranial recordings, the present data indicate that the chance of a good outcome with respect to seizure control deteriorates if there is more than one cavernoma, the distance between the dipole sites and the border of the lesion exceeds $2 \mathrm{~cm}$, or if functional considerations prevent the resection of the epileptogenic tissue outside the lesion proper.

One patient (no. 5) was treated with fractionated stereotactic radiotherapy, applied to the site of epileptogenicity indicated by MSI after previous resection of a cavernoma. In this case, where the removal of the cavernoma had no worthwhile effect upon seizure frequency, MSI revealed the extraordinary situation of non-lesional epileptogenicity in spite of the presence of a lesion and disclosed the real location of epileptogenic tissue. A similar finding was

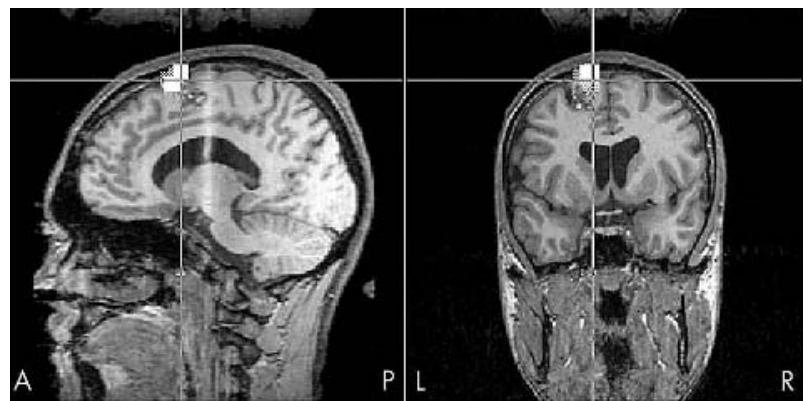

Figure 3 Preoperative MEG spike localisations at the border of lesion Source model: moving dipole; volume conductor model: realistic model (BEM). 


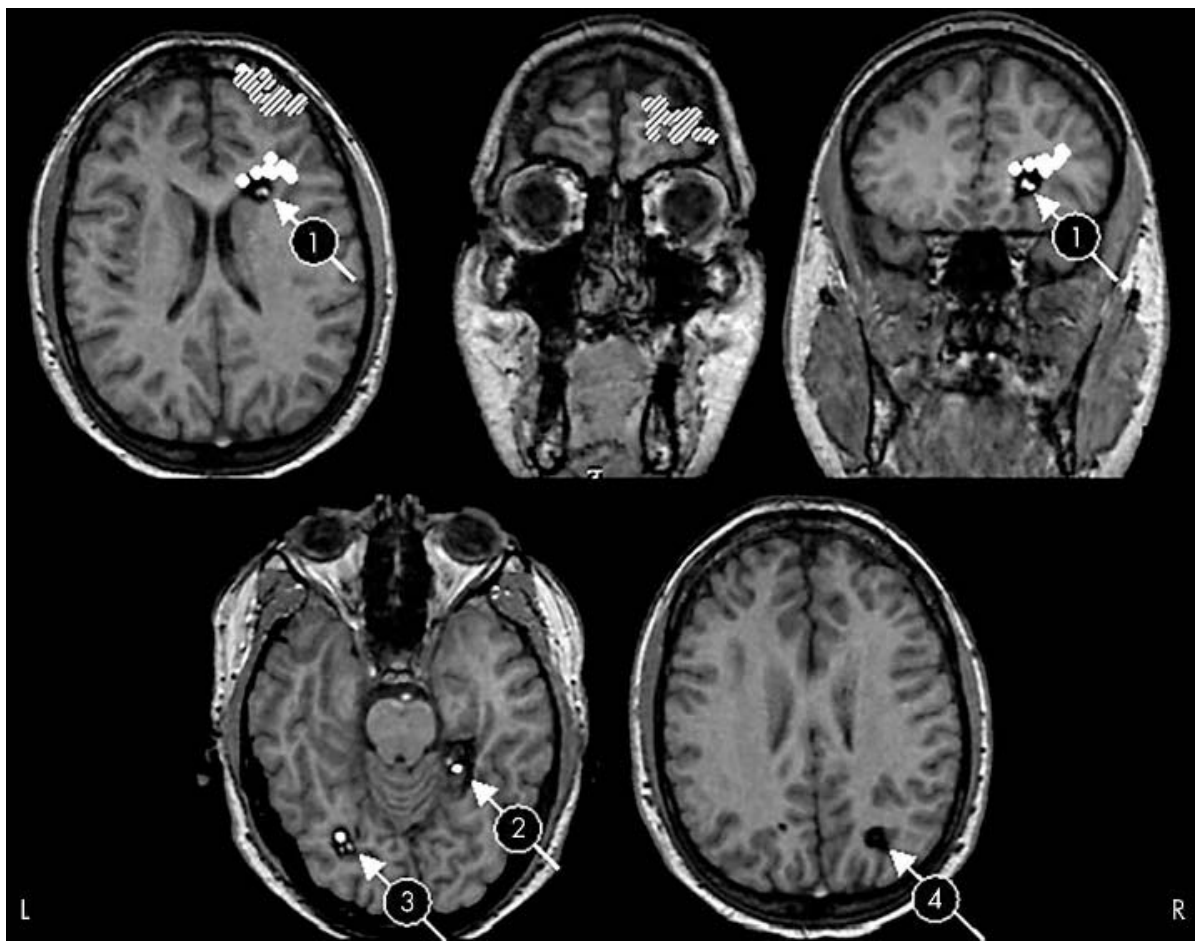

Figure 4 Patient with multiple cavernomas. Location of cavernomas (arrows) in the right frontal (1), right temporal (2), left occipital (3), and right occipital (4) lobes. Epileptic activity was found at the border of the frontal lesion (white areas) and, in addition, in the frontopolar region (hatched areas). Source model: moving dipole; volume conductor model: sphere.

observed in a case with four cavernomas identified by MRI scans, where MSI not only identified one of the multiple lesions as epileptogenic, but, furthermore, revealed an additional, non-lesional source of epileptic activity. Thus, in two cases MSI disclosed epileptogenic areas in otherwise inconspicuous sites remote from the known lesions. These areas were not identified by any other method.

Two patients, who had had previous surgical treatment, were undergoing additional therapeutic procedures, one case underwent second surgery and the other was the patient receiving radiotherapy. In both cases the treatment plan was guided by the MSI results. Taking into account the particular conditions of altered conductive properties as well as adherences after neurosurgery, MSI can be considered likely to be the most advantageous localising method for these cases, for, on the one hand, as a non-invasive tool, it is not impeded by adherences, nor on the other hand, by varying conductivities. EEG dipole source reconstructions, which have also been employed for localisations of lesional epileptic activity, ${ }^{26-28}$ may have failed here due to the liability of electric fields to be distorted by conductivity changes.

Six out of eight patients had monofocal MSI results. Thus, among four patients who had multiple cavernomas, two were identified by MSI to have monofocal epilepsy, with MSI indicating which of several lesions was the crucial one generating epileptic discharges. One of these patients was among the successfully operated group.

The conclusions for the surgical planning of epileptogenic cavernomas are as follows: in the case of focal seizures due to a single cavernoma, MEG may precisely delineate the epileptogenic tissue bordering the lesion, thus permitting appropriate inclusion of this specific area into the resection, instead of a plain lesionectomy which may not be sufficient for seizure control; and in patients with multiple cavernomas or dual pathology, MSI may reveal the complexity of the case and contribute to the decision about further invasive diagnostics and more sophisticated therapeutic measures.

The encouraging results of this paper should stimulate further studies with a larger number of cases, and with special attention to the assessment of the extent of the perilesional pathology-for example, by means of histological examination of the resected specimen and/or functional MRI.

\section{Authors' affiliations}

H Stefan, G Scheler, C Hummel, J Walter, Department of Neurology, Epilepsy and Neuro-Center, University of Erlangen-Nürnberg, Erlangen, Germany

J Romstöck, M Buchfelder, Department of Neurosurgery, University of Erlangen-Nürnberg, Erlangen, Germany

I Blümcke, Department of Neuropathology, University of ErlangenNürnberg, Erlangen, Germany

This study was supported by DFG grant STE-380/9-2.

Competing interests: none declared

\section{REFERENCES}

1 Zevgaridis D, van Velthoven V, Ebeling U, et al. Seizure control following surgery in supratentorial cavernous malformations: a retrospective study in 77 patients. Acta Neurochir (Wien) 1996;138:672-7.

2 Williamson A, Patrylo PR, Lee S, et al. Physiology of human cortical neurons adjacent to cavernous malformations and tumors. Epilepsia 2001;42(suppl 7):208-9.

3 Kirchberger K, Hummel C, Stefan H. Postoperative multichannel magnetoencephalography in patients with recurrent seizures after epilepsy surgery. Acta Neurol Scand 1998;98:1-7.

4 Pataraia E, Baumgartner C, Lindinger, et al. Magnetoencephalography in presurgical epilepsy evaluation. Neurosurg Rev 2002;25:141-59, discussion 160-1.

5 Iwasaki M, Nakasato N, Shamoto H, et al. Surgical implications of neuromagnetic spike localization in temporal lobe epilepsy. Epilepsia 2002;43:415-24.

6 Paetau R, Hämäläinen M, Hari R, et al. Magnetoencephalographic evaluation of children and adolescents with intractable epilepsy. Epilepsia 1994:35:275-84.

7 Stefan H. Clinical applications of MEG in epilepsy. Brain Topogr 1993;5:425-7

8 Stefan H, Schneider S, Fuchs K, et al. Magnetic source localization in focal epilepsy. Multichannel magnetoencephalography correlated with magnetic resonance brain imaging. Brain 1990;113(Pt 5):1347-59

9 Baumgartner C, Deecke L. Magnetoencephalography in clinical epileptology and epilepsy research. Brain Topogr 1990;2:203-19.

10 Mamelak AN, Lopez N, Akhtari M, et al. Magnetoencephalography-directed surgery in patients with neocortical epilepsy. J Neurosurg 2002;97:865-73.

11 Ebersole JS. Magnetoencephalography/magnetic source imaging in the assessment of patients with epilepsy. Epilepsia 1997;38(suppl 4): 1-5. 
12 Smith JR, Gallen C, Orrison W, et al. Role of multichannel magnetoencephalography in the evaluation of ablative seizure surgery candidates. Stereotact Funct Neurosurg 1994;62:238-44.

13 Hämäläinen M. Anatomical correlates for magnetoencephalography: integration with magnetic resonance images. Clin Phys Physiol Meas $1991 ; 12$ (suppl A):29-32.

14 Stefan H, Hummel C, Hopfengärtner R, et al. Magnetoencephalography in extratemporal epilepsy. J Clin Neurophysiol 2000;17:190-200.

15 Stefan H, Hummel C. Magnetoencephalography. In: Meinardi H, eds. Handbook of Clinical Neurology. Amsterdam: Elsevier, 1999:319-36.

16 Stefan H, Schneider S, Feistel H, et al. Ictal and interictal activity in partial epilepsy recorded with multichannel magnetoelectroencephalography: correlation of electroencephalography/electrocorticography, magnetic resonance imaging, single photon emission computed tomography, and positron emission tomography findings. Epilepsia 1992;33:874-87.

17 Tilz C, Hummel C, Kettenmann B, et al. Ictal onset localization of epileptic seizures by magnetoencephalography. Acta Neurol Scand 2002;106:190-5.

18 Awad IA, Rosenfeld J, Ahl J, et al. Intractable epilepsy and structural lesions of the brain: mapping, resection strategies, and seizure outcome. Epilepsia 1991;32:179-86.

19 Hattori H, Yamano T, Tsutada T, et al. Magnetoencephalography in the detection of focal lesions in West syndrome. Brain Dev 2001;23:528-32.

20 Hund M, Rezai AR, Kronberg E, et al. Magnetoencephalographic mapping basic of a new functional risk profile in the selection of patients with cortical brain lesions. Neurosurgery 1997;40:936-42.
21 Morioka T, Yamamoto T, Katsuta T, et al. Presurgical three-dimensional magnetic source imaging of the somatosensory cortex in a patient with a periRolandic lesion: technical note. Neurosurgery 1994;34:930-3.

22 Otsubo H, Ochi A, Elliott I, et al. MEG predicts epileptic zone in lesional extrahippocampal epilepsy: 12 pediatric surgery cases. Epilepsia 2001;42:1523-30

23 Ishibashi H, Simos PG, Wheless JW, et al. Localization of ictal and interictal bursting epileptogenic activity in focal cortical dysplasia: agreement of magnetoencephalography and electrocorticography. Neurol Res 2002;24:525-30.

24 Stefan H, Hummel C, Grabenbauer GG, et al. Successful treatment of focal epilepsy by fractionated stereotactic radiotherapy. Eur Neurol 1998;39:248-50.

25 Aung M, Sobel DF, Gallen CC, et al. Potential contribution of bilateral magnetic source imaging to the evaluation of epilepsy surgery candidates. Neurosurgery 1995; 37:1113-20.

26 Krings T, Chiappa KH, Cuffin BN, et al. Accuracy of electroencephalographic dipole localization of epileptiform activities associated with focal brain lesions. Ann Neurol 1998;44:76-86.

27 Ossenblok $P$, Fuchs $M$, Velis DN, et al. Source analysis of lesional frontal-lobe epilepsy. IEEE Eng Med Biol Mag 1999;18:67-77.

28 Sgouros S, Seri S, Natarajan K. The clinical value of electroencephalogram/ magnetic resonance imaging co-registration and three-dimensional reconstruction in the surgical treatment of epileptogenic lesions. Childs Nery Syst 2001;17:139-44.

\section{Call for papers}

10th European Forum on Quality Improvement in Health Care

13-15 April 2005, ExCel, Docklands, London

For further information on how to submit your paper please go to:

http://www.quality.bmipg.com 